\title{
Deregulated Expression of ANXA1 in Human High-Grade Breast Cancers
}

Einas Mohamed Yousef, David Laperrière, Muhammad Ramzan-Tahir, Sylvie Mader and Louis Arthur Gaboury* Institute for Research in Immunology and Cancer (IRIC), Montreal, Quebec, Canada

\begin{abstract}
Background: Breast cancer is a heterogeneous disease comprising a diversity of tumor subtypes. All of them differ from each other resulting in a wide array of clinical pictures, risks of recurrence and response to treatment. In recent years, tumor biomarkers have changed the way breast cancers are diagnosed and treated. In this paper we have sought to investigate the differential expression of ANXA1, a multifunctional calcium binding protein, among various molecular subtypes of breast cancers and in particular triple negative tumors.

Methods: ANXA1 was first studied using in-silico analysis on available DNA microarray and RNA sequencing data of human breast tissues. Next we ascertained ANXA1 expression on cell lines and breast carcinoma tissue microarrays along with cognate normal breast tissue.

Results: Whereas ANXA1 expression is normally restricted to the normal myoepithelial cell layer it becomes ectopically and aberrantly expressed in tumor cells of a significant minority of aggressive breast cancers. Specifically, we found that ANXA1 expression is severely deregulated in high-grade breast cancers that comprise clinically aggressive tumors such as triple-negative and, to some extent, HER2-positive breast cancers.

Conclusion: Our results indicate that ANXA1 is a valuable breast cancer biomarker that can help to segregate and dissect out subsets of high histological grade breast cancers paving the way to a better understanding of breast cancer progression and metastasis.
\end{abstract}

Keywords: ANXA1; Multifunctional calcium binding protein; Breast cancer

\section{Introduction}

Breast cancer is a heterogeneous disease characterized by a wide range of pathological features, diverse clinical behavior and variable response to treatment based on a complex interplay between characteristics of the tumor and the host [1]. In 2013, approximately 1 in 8 (12\%) women in the USA will develop invasive breast cancer. Breast cancer is the second cause of death after lung cancer among American women. The American Cancer Society's estimated 39,620 deaths from breast cancer patients to occur this year [2]. The high mortality rate from breast cancer results mainly from metastasis due to spread of cancer cells to distant organs such as the liver, lungs, brain and bones [3]. In selective subtypes of breast cancer, therapies targeting specific signaling pathways are well known and many of them are now widely used in the clinics [4]. For instance, hormonal therapy (Tamoxifen and aromatase inhibitors) can inhibit the effect of estrogen or decrease estrogen levels in patients with hormonally responsive tumors. In HER2-positive patients, Trastuzumab can prolong survival and lower the risk of relapse. Unfortunately, patients whose tumor fail to express hormonal receptors or lack HER2 overexpression will not benefit from those types of treatment and hence must rely mainly on chemotherapy. There is now a large body of evidence to suggest that cancer treatment must be tailored to individual tumor characteristics [5]. Despite the success of current therapies, we still need to uncover unique genetic alterations or tumor characteristics that might be translated into prognostic and predictive biomarkers or pharmacologically amenable targets Annexin A1 (ANXA1), the first characterized member of the annexin superfamily, is a calcium and phosopholipid binding protein known to mediate the anti-inflammatory actions of glucocorticoids [6]. However, it possesses many other functions that impact on key cellular processes such as proliferation [7], differentiation, cytoskeletal organization, cell migration [8], and apoptosis [9]. Expression of ANXA1 has been associated with the development and progression of different types of cancer. On the one hand, ANXA1 has been shown to be down-regulated in esophageal cancer [10], head and neck cancer
[11], and prostate cancer [12]. On the other, ANXA1 was found to be up-regulated in other types of malignancies such as pancreatic cancer [13], hepatocellular carcinoma [14] and stomach cancer [15].

We became interested in the status of ANXA1 gene expression in breast carcinomas following the seminal work by Perou et al. [16] on the molecular classes of breast cancer. Although the authors did not expand specifically on the putative role of ANXA1 in breast cancer, molecular profiling of tumors clearly indicated that ANXA1 was related to the basal-like subtype of breast cancer. Upon carefully reviewing the literature we found that the status of ANXA1 expression and its role in initiation and progression of breast cancer was still an unresolved issue. It was reported that a lower expression of ANXA1 was significantly associated with advanced stage of breast cancer and poor overall survival when compared to patients with high ANXA1 expression [17]. Likewise, it has also been shown that expression of ANXA1 is downregulated in metastatic tumors suggesting that ANXA1 functions as an Epithelial-Mesenchymal Transition (EMT) /metastatic suppressor [18]. These observations are hard to reconcile though with the findings by Yi and Schnitzer [19] who reported that ANXA1 null mice had impaired tumor growth and a lower propensity to develop metastasis. Furthermore, it was reported by others that ANXA1 promotes metastasis formation of basal-like breast cancer through its regulation of TGF- $\beta$ [20]. Indeed, Yom et al. [21] demonstrated that high ANXA1

*Corresponding author: Louis Arthur Gaboury, Institute for Research in Immunology and Cancer (IRIC) Montreal, Quebec, Canada, Tel: (514) 343-7770; Fax: (514) 343-7780; E-mail: louis.gaboury@umontreal.ca

Received October 09, 2013; Accepted November 27, 2013; Published November 29, 2013

Citation: Yousef EM, Laperrière D, Ramzan-Tahir M, Mader S, Gaboury LA (2013) Deregulated Expression of ANXA1 in Human High-Grade Breast Cancers. J Mol Biomark Diagn 4: 154. doi:10.4172/2155-9929.1000155

Copyright: (c) 2013 Yousef EM, et al. This is an open-access article distributed under the terms of the Creative Commons Attribution License, which permits unrestricted use, distribution, and reproduction in any medium, provided the original author and source are credited 
expression was significantly correlated with unfavorable prognostic factors such as hormone receptor negativity, HER2 overexpression and triple-negative breast cancer.

In this study, we investigated ANXA1 expression using in-silico analysis on available DNA microarray and RNA sequencing data of both normal human breast tissue and in a variety of human breast cancers. We also carried out immunohistochemical analyses on a large number of normal breast tissue and breast carcinoma using Cell Microarrays (CMA) and Tissue Microarrays (TMA). This allowed us to compare ANXA1 expression at the protein level with that of other available breast biomarkers. Here we show that ANXA1 is differentially expressed in normal human breast tissue and in breast tumors. Moreover, we found that ANXA1 expression is severely deregulated in high-grade breast cancers that comprise clinically aggressive tumors such as triplenegative and to some extent HER2-positive breast cancers. Our results suggest that ANXA1 is a valuable breast cancer biomarker whose deregulated pattern of expression may help to segregate subsets of high histological grade breast cancers such as triple-negative category and HER2-positive breast tumors into clinically meaningful categories.

\section{Materials and Methods}

\section{In-silico analysis}

The web application bc-GenExMiner [22] was used for correlation analysis of ANXA1 gene expression on a dataset comprising over 3400 microarrays. The "aov" and "TukeyHSD" functions were carried out to compare the mRNA levels within each breast cancer molecular subtypes for the ANOVA and Tukey multiple comparisons of means. The ANOVA was applied to check for an overall difference of expression level between molecular subtypes. The Tukey multiple comparisons of means were used to test for a significant difference between two subtypes (e.g. Luminal A vs. Basal). For both tests, a $p$-value $<0.05$ was considered significant. The mRNA levels of ANXA1, Vimentin (VIM), ESR1 and FOXA1 were compared using publically available microarrays and mRNA sequencing breast cancer patients datasets. The former included 51 breast cancer cell lines [23] and 247 patients [24]. The mRNA sequencing dataset from the Cancer Genome Atlas Network [25] included 844 patients. Moreover, MiSTIC dataset was also used to correlate ANXA1 with a different set of genes in normal breast tissue (102 cases) and breast cancer ( 756 patients) based on RNAsequencing data derived from The Cancer Genome Atlas (TCGA). Pearson correlation coefficient between set of genes was calculated with the "cor.test" function of the R language and environment for statistical computing (http://www.R-project.org/).

\section{Cell culture}

All cell lines were purchased from the American Type Culture Collection (ATCC). MCF10F and MDA-MB-231 cells were maintained at $37^{\circ} \mathrm{C}$ in Dulbecco's modified Eagle's medium Nutrient Mixture F-12 (DMEM/F12) (Wisent) supplemented with 10\% fetal bovine serum (FBS) (Sigma), $0.5 \mu \mathrm{g} / \mathrm{ml}$ hydrocortisone, $10 \mathrm{ng} / \mathrm{ml} \mathrm{EGF,} 10 \mu \mathrm{g} /$ $\mathrm{ml}$ insulin, $1 \%$ penicillin-streptomycin and $100 \mathrm{ng} / \mathrm{ml}$ choleratoxin (Wisent). 184-B5 cell line was maintained in the same medium except that $1 \mathrm{ng} / \mathrm{ml}$ of choleratoxin was used instead of $100 \mathrm{ng} / \mathrm{ml}$. MCF-7 cell line was maintained in Dulbecco's modified Eagle's medium Nutrient Mixture (DMEM) supplemented with 10\% FBS and 1\% penicillinstreptomycin. ZR-75-1 cell line was maintained in RPMI with $10 \%$ FBS and $1 \%$ penicillin-streptomycin. MDA-MB-361 was maintained in L15+2 mM Glutamine+15\% FBS. MDA-MB-468 was maintained in Ham's-F12 medium (1:1 mixture) supplemented with $2 \mathrm{mM}$ L-glutamine and 5\% FBS.

\section{Preparation of paraffin-embedded cell pellets}

All cell lines were maintained in culture until they reached $80 \%$ confluence. Petri dishes were then washed with PBS followed by trypsinization of the cell layer until they completely detached from the bottom of the dish, then media specific for each cell line was added to inactivate trypsin. Cells and media were collected in $15 \mathrm{ml}$ tube and centrifuged at $1000 \mathrm{rpm}$ for 5 minutes. The supernatant was aspirated and the pellets were resuspended in $10 \%$ neutral buffered formalin and kept at $4^{\circ} \mathrm{C}$ for 24 hours to allow proper fixation. After 24 hours, the cells were centrifuged at $1,800 \mathrm{rpm}$ for 10 minutes and the supernatant was aspirated and the pellets resuspended in melted HistoGel (thermoscientific). Cell pellets were maintained in HistoGel at $4^{\circ} \mathrm{C}$ for 20 minutes until the gel solidified. Pellets were then removed from the eppendorf tubes and placed in embedding cassettes for further fixation in 10\% neutral buffered formalin. Cell pellets were next processed in SAKURA Tissue-Tek VIP (Vacuum infiltration processor) then embedded in paraffin blocks. $3 \mu \mathrm{m}$ section from each block was prepared and stained with Haematoxylin and Eosin (HandE) to evaluate the relative position of each cell cluster in each block and to determine cellularity.

\section{Cell microarray (CMA) construction}

CMA was prepared as follows: two punches $(2 \mathrm{~mm})$ from each cell line were plucked and inserted into recipient blocks according to the intended design of the map using a Manual Tissue Arrayer I (Beecher Instruments). Blocks were next inverted and incubated overnight in the oven over a glass slide. The blocks were allowed to cool off until they could be detached from the glass slide. $3 \mu \mathrm{m}$ sections were prepared from the CMA and stained with HandE or submitted to automated immunohistochemical reactions (IHC).

\section{Patient samples and tissue microarray (TMA) construction}

This study was conducted on 300 archived Formalin-Fixed, ParaffinEmbedded (FFPE) samples containing both normal and tumor tissues obtained from female patients. All samples were obtained from Centre Hospitalier de l'Université de Montréal (CHUM) after obtaining the approval of the research ethical committee (SL 05.019). The collected blocks contained both invasive and in situ carcinoma of the breast obtained at surgery (lumpectomy or mastectomy). Normal breast tissues were obtained from healthy women undergoing plastic surgery (Table 1). A number of extraneous tissues such as colon, thyroid and placenta were included in each TMA to serve as external controls.

\begin{tabular}{|l|c|c|}
\hline Variables & No. of cores & $\%$ \\
\hline Organs used in TMA & 643 & \\
\hline Colon & 4 & 0.7 \\
\hline Lymph node & 26 & 4.0 \\
\hline Mammary gland & 596 & 92.7 \\
\hline Rectum & 11 & 1.7 \\
\hline Thyroid & 6 & 0.9 \\
\hline Grade & 570 & \\
\hline I & 54 & 9.5 \\
\hline II & 116 & 20.3 \\
\hline III & 400 & 70.2 \\
\hline Molecular subtypes & 488 & \\
\hline Luminal A & 143 & 29.3 \\
\hline Luminal B & 54 & 11.1 \\
\hline HER2-positive & 68 & 13.9 \\
\hline Triple-negative & 223 & 45.7 \\
\hline
\end{tabular}

Table 1: Clinico-pathological data of tissues used in TMAs. 
To construct TMAs, $4 \mu \mathrm{m}$ sections from each paraffin donor block were stained with HandE. These sections were microscopically examined by two independent pathologists to select the most representative fields. Areas from each corresponding paraffin blocks were plucked in duplicate or triplicate and $1 \mathrm{~mm}$ core punches realigned into recipient blocks using a Manual Tissue Arrayer I (Beecher Instruments). Tissue sections from each TMA were prepared and one slide from each block was stained with HandE to review the diagnoses and histological grades on all tissue samples.

\section{Histological grading of breast cancer}

Modified Scarff-Bloom-Richardson-Ellis-Elston grading system (SBR-EE) [26] was used to score invasive breast cancers. The patients were classified into one of three different histological grades (Grade I, II and III). Briefly, tumor grade is determined based on three parameters:extent of tubule formation, mitotic rate and nuclear size. Each of these features is assigned a score ranging from 1-3. Next, the scores for each criterion are added together with a cumulative figure ranging from 3 to 9. Grade I tumors are well differentiated (low grade) with a total score of 3 to 5 . Grade II tumors are moderately differentiated (intermediate grade) with a total score of 6 to 7. Grade III are poorly differentiated (high grade) with a total score of 8 to 9 .

\section{Immunohistochemistry}

Immunohistochemistry was carried out according to manufacturer recommendations on an automated immunostainer (Discovery XT system, Ventana Medical Systems, Tucson, AZ). Antigen retrieval was performed with proprietary reagents followed by incubation with the primary antibody. Antibodies used are listed in (Table 2). Sections were then incubated with a specific secondary biotinylated antibody for 32 minutes. Streptavidin horseradish peroxidase, and 3,3-diaminobenzidine were used according to the manufacturer's instructions (DABmap detection kit, Ventana Medical Systems). Finally, sections were counterstained with Gill hematoxylin and sodium bicarbonate. For ANXA1, MDA-MB-231 cell line and thyroid carcinomas were used as positive controls while MCF-7 and ZR-75-1 cell lines served as negative controls. Each section was scanned at a high resolution (40X) using the Nanozoomer Digital Pathology equipment (Hamamatsu, Bridgewater, NJ).

\section{Scoring of stained slides}

Estrogen receptor (ER) and Progesterone Receptor (PR) status was determined using Allred's method [27]. Scoring of HER-2 was carried out according to the College of American Pathologists (CAP)approved scoring system [28]. Scoring of ANXA1, Ki-67, FOXA1 and VIM expression on each core was carried out using two tier scoring system. We first determined the percentage of immunoreactive cells (quantity score) which was estimated as follows (no staining was scored as $0,1-10 \%$ of cells with positive staining were scored as $1,10-50 \%$ as $2,50-70 \%$ as 3 , and $70-100 \%$ as 4 ). Next, we assessed the second parameter corresponding to the staining intensity (staining intensity score). The latter was rated as follows (No staining (negative) $\rightarrow 0$, weak staining $\rightarrow 1$, moderate staining $\rightarrow 2$, and strong staining $\rightarrow 3$ ). The immunohistochemical score was calculated by multiplying the quantity and staining intensity scores; the combined score could therefore range from 0 to 12 . An IHS score of 0 to 4 was considered to represent low expression while scores more than 4 to 12 were considered high expression [13]. The criteria used to define molecular subgroups of breast cancer are listed in (Table 3) [29].

\begin{tabular}{|c|c|c|c|c|c|}
\hline Antibody & Clone & Dilution & Provider & $\begin{array}{l}\text { Retrieval } \\
\text { method }\end{array}$ & Positive score \\
\hline ER & SP1 & Ready to use & Ventana & HIER pH 6 & Allred score $\geq 3$ \\
\hline PR & 1E2 & Ready to use & Ventana & HIER pH 6 & Allred score $\geq 3$ \\
\hline HER2 & 4B5 & Ready to use & Ventana & HIER pH 6 & $3+$ \\
\hline Ki-67 & SP6 & $1 / 100$ & BioCare & HIER pH 6 & $>14 \%$ \\
\hline ANXA1 & Clone29 & $1 / 500$ & BD & HIER pH 9 & $\begin{array}{l}\text { 0-4 low expression } \\
6-12 \text { high expression }\end{array}$ \\
\hline FOXA1 & $\begin{array}{l}\text { Poly- } \\
\text { clonal }\end{array}$ & $1 / 500$ & Abcam & HIER pH 6 & $\begin{array}{l}\text { 0-4 low expression } \\
6-12 \text { high expression }\end{array}$ \\
\hline VIM & 3B4 & $1 / 100$ & Dako & HIER pH 6 & $\begin{array}{l}\text { 0-4 low expression } \\
5-12 \text { high expression }\end{array}$ \\
\hline
\end{tabular}

ER: Estrogen receptor; PR: Progesterone receptor; HER2: Human epidermal growth factor receptor 2; ANXA1, Annexin A1; FOXA1: Forkhead box protein A1: VIM: Vimentin; HIER: Heat-induced epitope retrieval

Table 2: Antibodies used for immunohistochemistry.

\begin{tabular}{|l|c|c|l|l|}
\hline Molecular subtypes & ER & PR & HER-2 & Ki-67 \\
\hline Luminal A & + & + & - & $<14 \%$ \\
\hline Luminal B (1) & + & + & + & \\
\hline Luminal B (2) & + & + & - & $\geq 14 \%$ \\
\hline HER-2 positive & - & - & + & \\
\hline Triple negative & - & - & - & \\
\hline
\end{tabular}

Table 3: Criteria used to define molecular subtypes of breast cancer.

\section{Immunofluorescence and confocal microscopy}

Direct immunofluorescence staining was carried out using Ventana automated immunostainer. Dylights 550 and 650 fluorophores from Thermo Scientific Pierce protein biology products were used to label primary antibodies against p63 (p63 Ab-1; IgG clone 4A4, Thermoscientific) and ANXA1 (ANXA1; IgG1 clone29/Annexin I, BD transduction laboratory). The slides were incubated with the labeled antibodies for six hours. The slides were next washed and DAPI was applied. Sections were cover slipped and kept at $-20^{\circ} \mathrm{C}$.

Fluorescent images were captured using Zeiss LSM 510 laser scanning confocal microscope equipped with $63 \mathrm{x}$ oil immersion objective lens. META detector was used for spectral separation of fluorochromes with overlapping spectra. Images were cropped, resized, and brightness and contrast over the whole image adjusted where necessary, using AxioVision and ImageJ softwares.

\section{Statistical analyses}

All statistical analyses were carried out using different packages of language R. The distribution of ANXA1 in different histological grades and molecular subtypes is shown using box plots and bar charts. Non-parametric tests are used due to the nature of ordinal and categorical data. The overall relationship between ANXA1 scores and other characteristics (i.e. histological grades, and molecular subtypes) was evaluated by chi-square test. Correlation analysis for immunohistochemical expression levels was carried out using the Spearman's rho correlation coefficient. Statistical significance was considered, with a $p$-value $<0.05$.

\section{Results}

ANXA1 is highly expressed in basal-like breast cancer- insilico analysis

Comparison of mRNA levels within breast cancer molecular subtypes using the bc-GenExMiner database comprising over 3400 microarrays and the mRNA sequencing dataset from the Cancer 
Citation: Yousef EM, Laperrière D, Ramzan-Tahir M, Mader S, Gaboury LA (2013) Deregulated Expression of ANXA1 in Human High-Grade Breast Cancers. J Mol Biomark Diagn 4: 155. doi:10.4172/2155-9929.1000155

ANXA1 mRNA Expression in PAM50 molecular subtypes

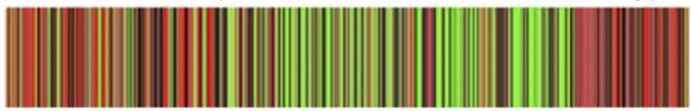

Low Expression

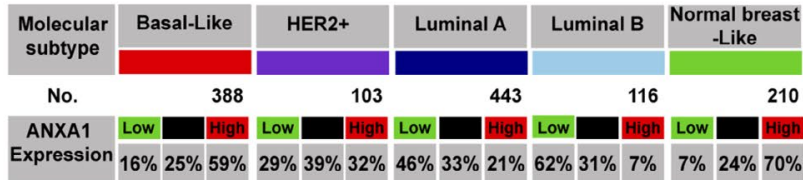

A

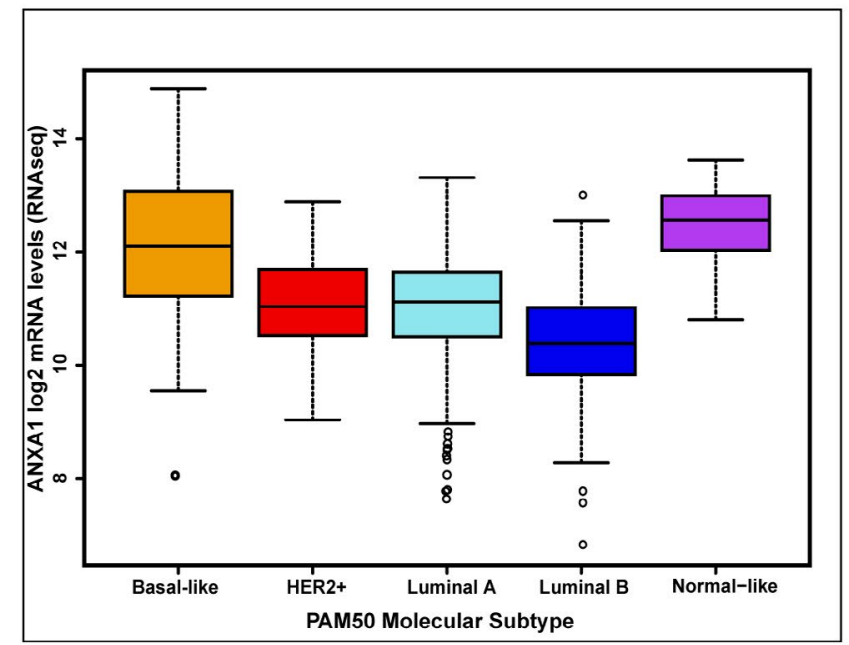

B

Figure 1: ANXA1 mRNA expression in PAM50 molecular subtypes of breast cancer using bc-GenExMiner database v3.0. A. The figure and table show the patients with low, intermediate and high ANXA1 expression in each molecular subtype. The basal-like and normal-like subtypes contain more patients with high expression of ANXA1. In contrast, luminal $A$ and $B$ subtypes comprise more patients with low expression of ANXA1. B. TCGA mRNA sequencing dataset including 844 patients showing that ANXA1 is highly expressed in basal-like subtype. ${ }^{* * *} p$-value $<0.001$, Tukey's test.

Genome Atlas (TCGA) dataset that included 844 patients were carried out. In patient microarray data sets, $59 \%$ of the basal-like and $70 \%$ of normal-like breast cancer subtypes showed high level of ANXA1 expression. In contrast, $21 \%$ of luminal A and only $7 \%$ of luminal B subtypes demonstrated high expression of ANXA1. For the HER2positive subtype, the percentage of patients with high or low expression of ANXA1 was nearly identical (32\% vs. $29 \%$, Figure 1A). Similarly, in the patient sequenced transcriptomes, ANXA1 mRNA levels were found to be significantly overexpressed in the basal-like subtype where compared to both luminal A $(p$-value $<0.001)$ and B subtypes (p-value $<0.001)$ (Figure 1B).

\section{ANXA1 is overexpressed in triple-negative breast cancer cell lines}

To validate the results obtained from our in-silico analysis, ANXA1 expression was studied in a panel of human breast cancer cell lines with varying degree of expression of ESR1, PR and HER2 (Table 4). ANXA1 expression was detected in all basal-like cell lines 184-B5, MCF10F, MDA-MB-468 and MDB-MB-231 [30]. Intense membrane staining was present in all triple-negative cell lines. However, cytoplasmic and nuclear staining intensity somewhat varied in these cell lines (Figure 2A-D). In sharp contrast, ANXA1 was absent from MCF7, ZR-75-1 and MDA-MB-361 cell lines (Figure 2A-F) which are all considered to belong to the luminal subtype categories $[30,31]$. Taken together, our findings in breast cancer cell lines support our in-silico analysis showing higher expression of ANXA1 in triple-negative breast cancer.

ANXA1 is strictly confined to myoepithelial cells in both normal breast tissue and in-situ breast cancers

In normal breast tissue and in-situ carcinoma, we found ANXA1 to be strongly expressed in myoepithelial cells and in a few adjacent stromal cells (Figure 3A and B). Myoepithelial cell identity was confirmed by their typical localization around the luminal cells, their characteristic flattened morphology and more directly by the coexpression of ANXA1 with the specific myoepithelial cell marker p63 using confocal microscopy. ANXA1 and p63 were co-localized in the very same myoepithelial cells either in normal mammary gland or in ductal carcinoma in situ (Figure 3C and D).

ANXA1 is overexpressed in subsets of triple-negative and HER2-positive breast cancers

Variation in the levels of ANXA1 expression according to molecular

\begin{tabular}{|l|c|c|c|c|c|}
\hline Cell Lines & ER & PR & HER2 & Molecular Subtypes & ANXA1 \\
\hline 184B5 & - & - & - & Basal & + \\
\hline MDA-MB-468 & - & - & - & Basal & + \\
\hline MCF10F & - & - & - & Basal & + \\
\hline MDAMB231 & - & - & - & Basal & + \\
\hline ZR-75-1 & + & + & - & Luminal & - \\
\hline MDA-MB-361 & + & - & + & Luminal & - \\
\hline MCF7 & + & + & - & Luminal & - \\
\hline
\end{tabular}

ER: Estrogen receptor; PR: Progesterone Receptor; HER2: Human Epidermal Growth Factor Receptor 2; ANXA1: Annexin A1

Table 4: Expression of ER, PR, HER2 and ANXA1 in different types of breast cancer cell lines.

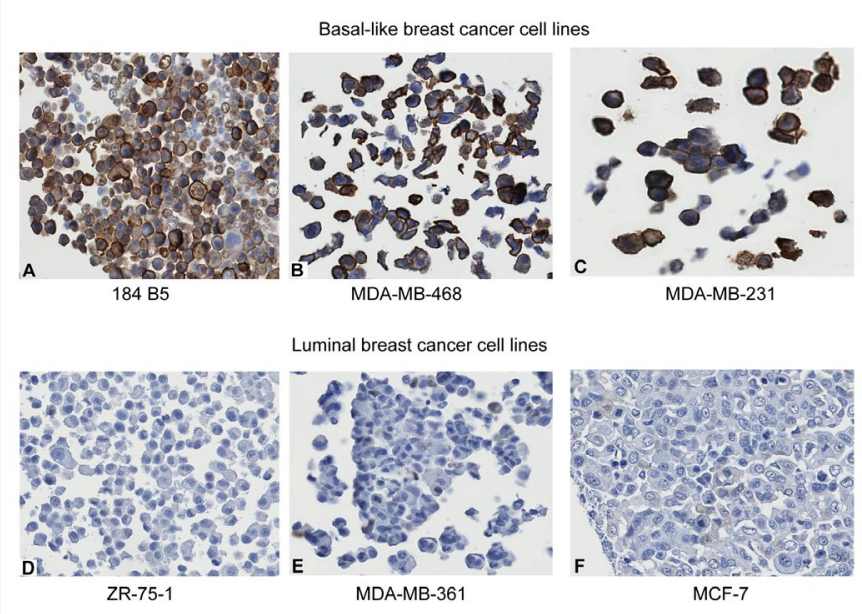

Figure 2: Expression of ANXA1 in different types of breast cancer cell lines. All basal-like breast cancer cell lines are positive for ANXA1 expression such as A. 184 B5, B. MDA-MB-468, C. MDA-MB-231. In contrast, in all luminal subtype cell lines ANXA1 is lacking such as D. ZR-75-1, E. MDA-MB-361 and F. MCF-7. Magnification: $40 \mathrm{X}(\mathrm{A}-\mathrm{F})$. 
Citation: Yousef EM, Laperrière D, Ramzan-Tahir M, Mader S, Gaboury LA (2013) Deregulated Expression of ANXA1 in Human High-Grade Breast Cancers. J Mol Biomark Diagn 4: 155. doi:10.4172/2155-9929.1000155

Page 5 of 10
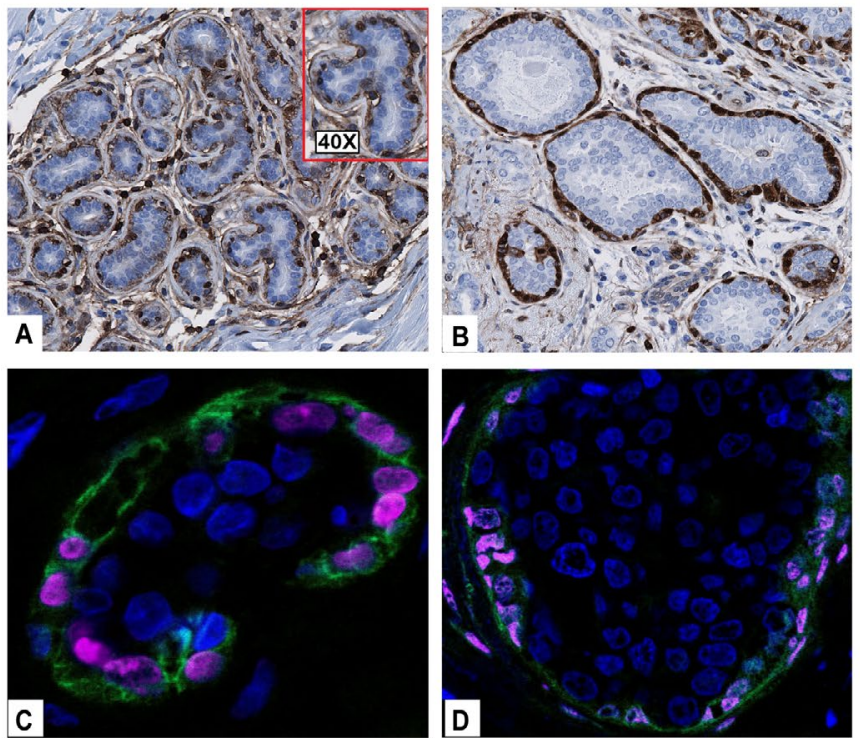

Figure 3: Expression of ANXA1 in normal breast tissue and in-situ carcinoma. A. Normal lobules displaying ANXA1 expression in myoepithelial cells and in a few stromal cells while luminal cells lack ANXA1 expression. B. DCIS shows a similar distribution of ANXA1 as that present in normal lobules. C. Co-localization of ANXA1 (cytoplasmic) and p63 (nuclear) in myoepithelial cells lining normal breast ducts using direct immunofluorescence and confocal microscopy. D. Colocalization of ANXA1 and p63 in myoepithelial cells of DCIS. Magnification: 20X $(A \& B), 40 X$ inset in Figure $A, 63 X(C \& D)$.

subtypes of breast cancer was assessed. All samples collected from patients with luminal A and luminal B breast cancer lacked detectable expression of ANXA1 (Figure 4A and B). In contrast, almost half of the samples collected from patients with triple-negative breast cancers had strikingly elevated levels of ANXA1 expression (Figure 4C and D). Moreover, the association of ANXA1 with basal-like breast cancer, which is considered to be a subset of triple-negative breast cancer, was further substantiated by a positive correlation with basal cytokeratin CK5/6 ( $r=0.72, p$-value $<0.001$ ) (data not included). Notably, most but not all HER2-positive samples (approx. 90\%) were found to be negative for ANXA1 (Figure 4E,F and G).

\section{Altered pattern of ANXA1 expression correlates with a subset of high-grade breast cancers.}

In Grade I, Grade II and $70.4 \%$ of Grade III breast cancer, ANXA1 expression could not be detected in tumor cells (Figure 5AC). However, $29.6 \%$ of Grade III breast cancer patients showed ectopic expression of ANXA1 in tumor cells (Figure 5D-G). Another key finding was the disordered cellular distribution of ANXA1 labeling in high-grade breast cancers. Whereas ANXA1 expression is normally restricted to the myoepithelial cells in a typically uniform fashion, the pattern observed in tumor cells markedly departed from that of normal cognate cells. Indeed, the staining pattern in tumors is at odds with that observed in normal myoepithelial cells. Some tumors exhibited ANXA1 staining mainly in the cytoplasm with only a few cells harboring nuclear staining. Other tumors demonstrated predominantly nuclear staining with only very faint cytoplasmic staining. On occasion, we have observed an intense nuclear and cytoplasmic staining. Lastly, strong membranous staining along with weak cytoplasmic staining was found in few high-grade breast cancers (Figure 5D-G). Taken together, our results indicated that not only ANXA1 was aberrantly accumulating in a significant minority of high grade tumor cells but also that there was an accompanying defect in its cellular translocation, distribution and compartmentalization

\section{Positive correlation between ANXA1 and vimentin and negative correlation between ANXA1, ESR1 and FOXA1.}

The RNA-sequencing data derived from TCGA dataset [23,32] was analysed using the MiSTIC visualization tool to identify genes that are positively or negatively correlated with ANXA1 in normal breast tissue
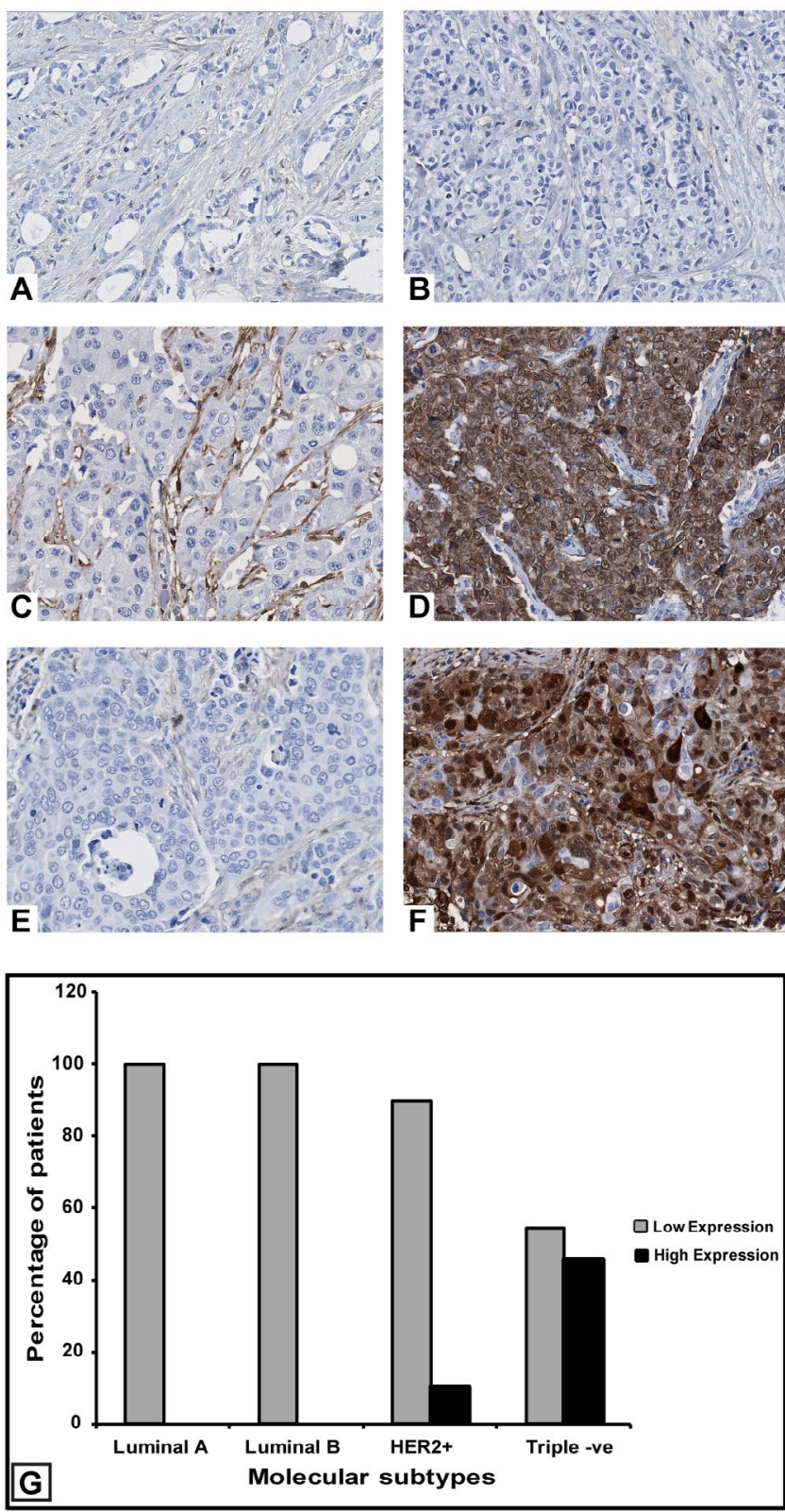

Figure 4: Expression of ANXA1 in different molecular subtypes of breast cancer A. Luminal A B. luminal B breast cancer fail to express ANXA1 in cancer cells. C and $D$. Triple-negative breast cancer. Some patients display negative or low level of ANXA1 expression (C) others showed high ANXA1 expression (D). E and F. HER2-positive breast cancers with either negative $(E)$ or positive expression $(F)$ of ANXA1. E. Histogram displaying the percentage of patients with high and low expression of ANXA1 in each molecular subtype of breast cancer. Magnification: $20 \mathrm{X}(\mathrm{A}-\mathrm{F})$. 


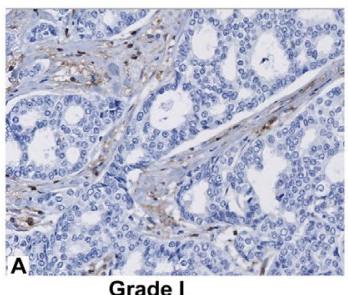

Grade I
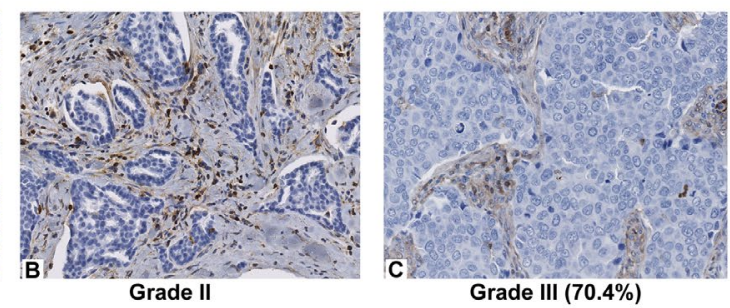

Grade III $(70.4 \%)$

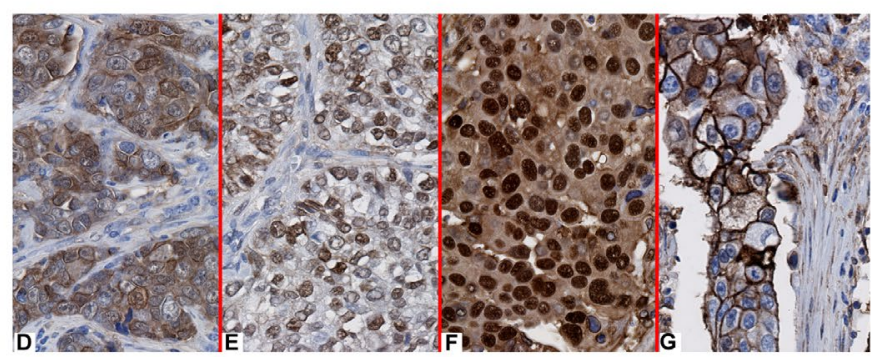

Grade III (29.6\%)

Figure 5: ANXA1 expression of breast cancer of different histological grades; A, B, C. Expression of ANXA1 is absent in Grade I, Grade II and some of Grade III breast cancer cells. Stromal cells are faintly positive for ANXA1. D-G. $29.6 \%$ of Grade III breast cancers have an altered pattern of ANXA1 expression along with varied subcellular localization. ANXA1 staining could be found in the cytoplasm (D), in the nuclei with only very faint cytoplasmic staining (E), in both cytoplasm and nuclei $(\mathrm{F})$, or in the membrane $(\mathrm{G})$ of breast cancer cells.

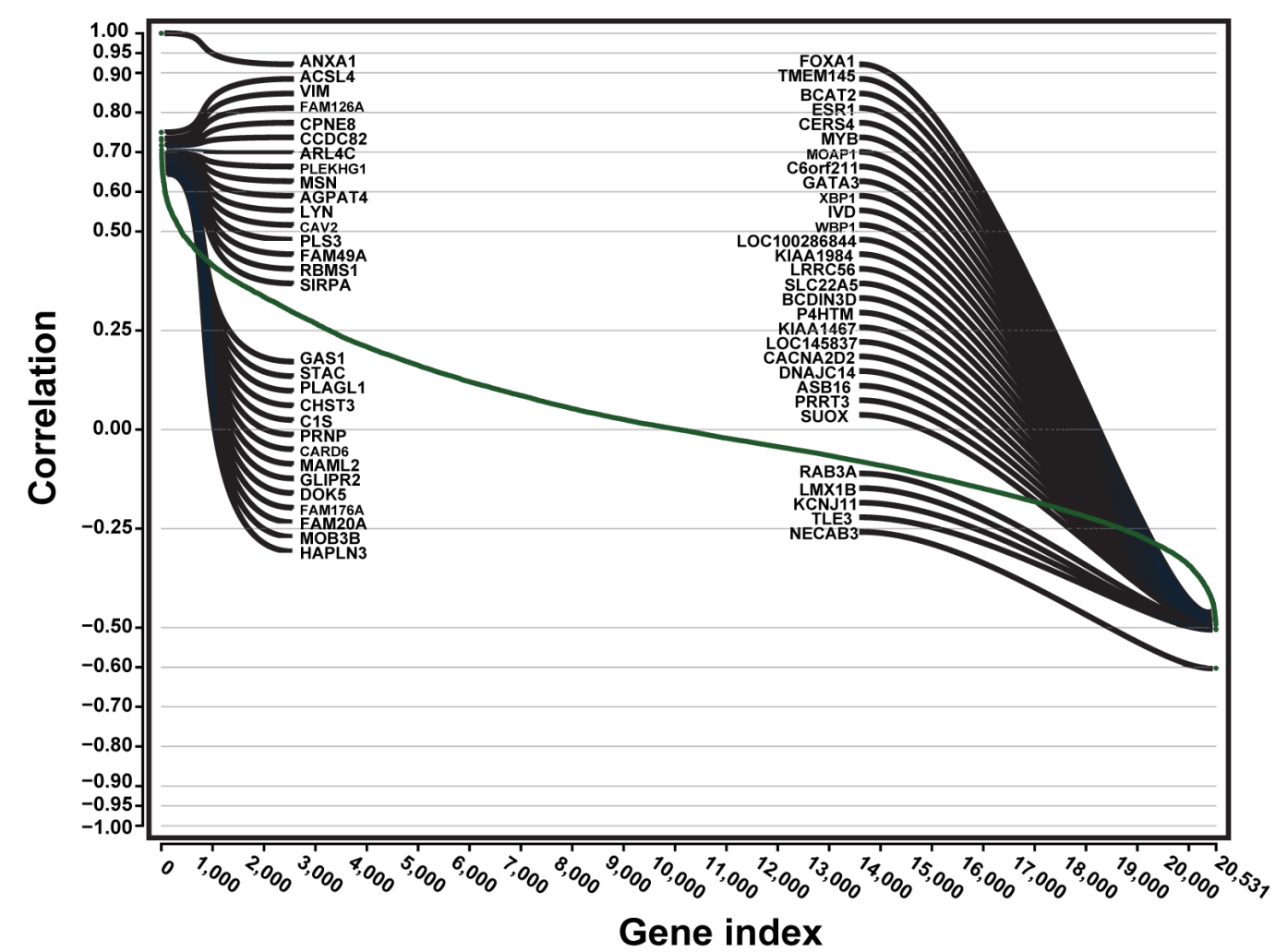

Figure 6: Genes that are positively or negatively correlated with ANXA1 in 756 breast cancer patients based on RNA-sequencing data derived from TCGA dataset. The list on the left side depicts the genes which are positively correlated with ANXA1 while those that appear on the right side are the genes that are negatively correlated with ANXA1.

(102 cases) and in breast cancer (756 patients). A positive correlation was observed between ANXA1 and VIM, one of the EMT markers in breast cancers $(r=0.73)$. In the same group of tumors, ANXA1 was negatively correlated with several luminal markers such as FOXA1 $(r=0.66)$, ESR1 $(r=0.46)$, GATA3 $(r=-0.47)$ and XBP1 $(r=-0.47)$ (Figure $6)$. To substantiate these observations, we next assessed the mRNA 


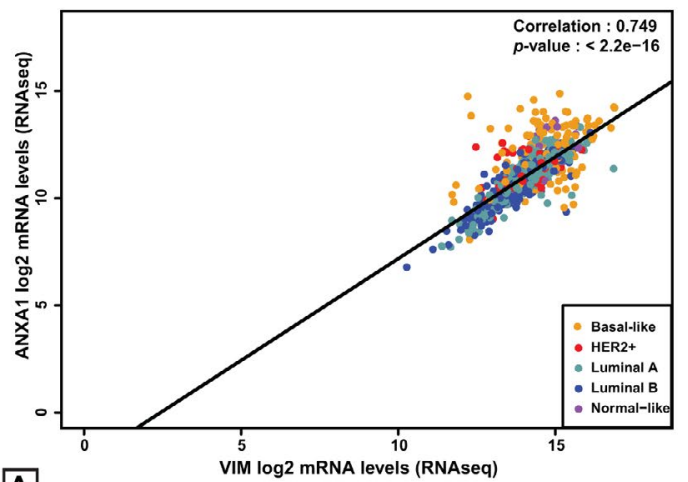

A
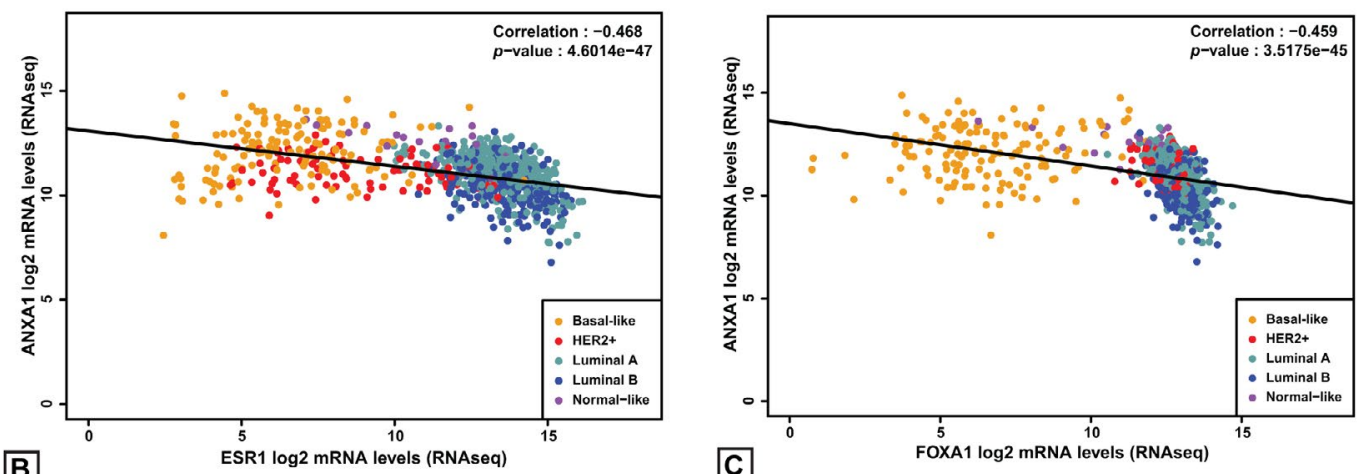

C

Figure 7: Correlation between ANXA1 and VIM, ESR1 and FOXA1 using RNA sequencing data of 844 breast cancer patients. A. Scatter plot of ANXA1 and VIM mRNA expression. There is a positive correlation between ANXA1 and VIM mRNA transcripts levels (Pearson's correlation analysis $n=844, r=0.749, p$-value $=2.2 \mathrm{e}-16$ two-sided). B. Scatter plot of ANXA1 and ESR1 mRNA expression. There is a negative correlation between ANXA1 and ESR1 mRNA transcripts levels (Pearson's correlation analysis $n=844, r=-0.468, p$-value $=4.6014 \mathrm{e}-47$ two-sided). C. Scatter plot of ANXA1 and FOXA1 mRNA expression. There is a negative correlation between ANXA1 and FOXA1 mRNA transcripts levels (Pearson's correlation analysis $n=844, r=-0.459$, p-value $=3.5175 e-45$ two-sided).

levels of ANXA1, VIM, ESR1 and FOXA1 using publically available microarrays and mRNA sequencing breast cancer patients datasets which included 51 breast cancer cell lines [23] and 247 patients [24]. Similar correlations could be established using mRNA in sequenced transcriptome datasets strengthening the above mentioned association. Specifically, a positive correlation between ANXA1 and VIM was detected $(\mathrm{r}=0.75$ Figure $7 \mathrm{~A})$ while a negative correlation was established between ANXA1 and ESR1 ( $\mathrm{r}=-0.47$ Figure 7B) and between ANXA1 and FOXA1 ( $r=-0.46$ Figure $7 \mathrm{C})$.

In order to expand on the data obtained from the in-silico analyses, we used additional sections of our human breast cancer TMAs to ascertain the expression of VIM, ESR1 and FOXA1 at the protein level. Again, a positive correlation between ANXA1 and VIM was confirmed $(r=0.48, p$-value $<0.001)$. In triple-negative breast cancer, $62 \%$ of patients had a high expression of both proteins. In contrast, 100 $\%$ of luminal A patients and $95.6 \%$ of luminal B had low expression of ANXA1 and VIM. Similarly, 77.3\% of HER2-positive patients also had low expression of ANXA1 and VIM with only $4.6 \%$ showed high expression of both proteins.

We were also able to confirm the negative correlation between ANXA1 and ESR1 $(r=-0.45, p$-value $<0.001)$ and between ANXA1 and FOXA1 ( $r=-0.51, p$-value $<0.001)$ using TMAs. Specifically, in luminal AandB breast cancer, $100 \%$ of the patients had a high degree of expression of ESR1 and FOXA1 and no detectable levels of ANXA1 expression (Figure 8A). In triple-negative breast cancer where ESR1 and FOXA1 are negative, $45.5 \%$ of patients had high levels of ANXA1 (Figure 8B).

\section{Discussion}

ANXA1 belongs to a family of calcium and phospholipid binding proteins initially considered to have an anti-inflammatory function. However, many recent publications also stressed its role in key cellular processes such as proliferation, differentiation, cytoskeletal organization, cell migration and apoptosis. Although ANXA1 expression has been studied in different types of cancer, so far results have been rather conflicting, with reports in the literature describing variable levels of expression in normal and breast cancer tissue. This also holds true for breast cancer where the status of ANXA1 expression in normal mammary gland and breast cancers is currently unsettled. In the present work, we have investigated the expression of ANXA1 in human breast cancer tissues. We found that deregulated ANXA1 expression is observed only in high-grade breast cancers that comprise clinically aggressive tumors such as triple-negative and HER2-positive breast cancers supporting the view that ANXA1 is a valuable marker for a subset of aggressive breast tumors.

Using TMAs comprising normal breast tissue and breast carcinomas of various histological grades, we conclusively demonstrated that 


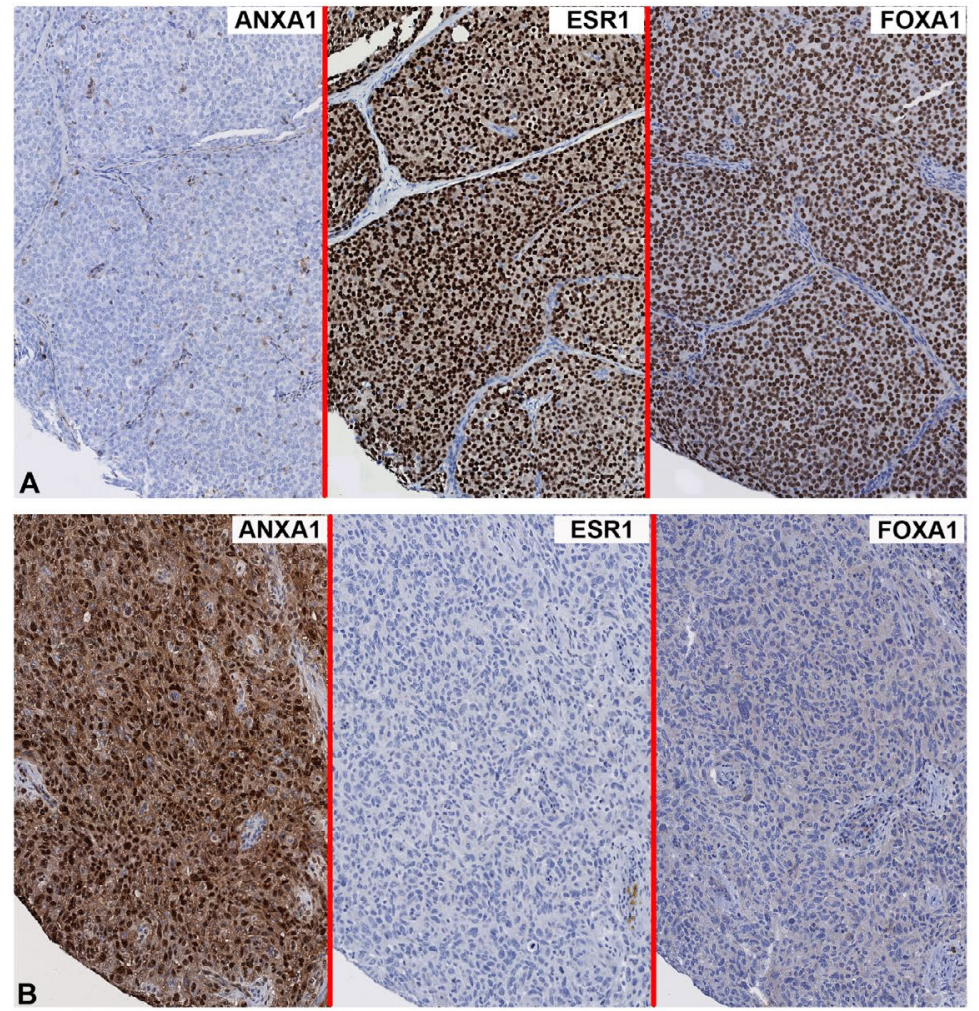

Figure 8: Correlation between ANXA1, ESR1 and FOXA1 in different molecular subtypes of breast cancer. A) Luminal breast cancers which have high expression of both ESR1 and FOXA1 fail to express ANAX1 B) 43\% of triple-negative breast cancer that are ESR1 and FOXA1 negative, display high levels of ANXA1. Magnification: $10 X(A-C)$.

ANXA1 expression is confined to the myoepithelial cell layer and to a few adjacent stromal cells of normal breast tissue. Myoepithelial cell identity was confirmed by their typical localization around the luminal cells, their characteristic flattened morphology and more directly by the co-expression of ANXA1 with the specific myoepithelial cell marker p63 using confocal microscopy. This constitutive pattern of ANXA1 expression is also highly conserved in the myoepithelial cell layer surrounding the ducts and lobules of in-situ carcinomas. Notably, there is a complete absence of ANXA1 in malignant epithelial cells where the normal regulatory pathways continue to operate. These results are consistent with those of Ang et al. [7] who reported similar pattern of expression of ANXA1 in myoepithelial cells. However, they are clearly at odds with the observations of Elshimali and Lui [33] who found that ANXA1 is expressed in both epithelial and myoepithelial cells of normal breast tissue.

Since there is complete disappearance of the myoepithelial cells layer during stromal infiltration, the lack of ANXA1 positive myoepithelial cells was an expected finding. This is consistent with the inability to detect ANXA1 in low-grade (Grade I) and intermediate-grade (Grade II) and $70.4 \%$ of high-grade (Grade III) breast cancer. Strikingly however, $29.6 \%$ of Grade III breast cancer patients showed ectopic expression of ANXA1 in tumor cells. The morphology of tumor cells and the failure to express any of the other myoepithelial cell markers are inconsistent with the possibility that those cells represent a residual myoepithelial population. Although it is tempting to hypothesize that positive tumor cells are derived from a progenitor cell endowed with both luminal and myoepithelial features, this view nevertheless fails to explain the absence of ANXA1 positive tumor cells in both grade
I and grade II tumors. In our opinion, ANXA1 expression in tumor cells more likely represents an abnormal, deregulated cellular process due to a failure of the checks and balances that operates under normal conditions in the breast tissue.

Our results on human breast cancer cell lines indicate that triplenegative cell lines expressed ANXA1 at significantly higher levels than those observed in both luminal A or B cell lines. This result is also consistent with the observations of Kang et al. [34] that breast cancer cell lines with high expression of ANXA1 are invasive by nature, whereas lines with low or undetectable expression of ANXA1 had a poor capacity for migration. Additionally, we found that enhanced expression of ANXA1 is a characteristic of a subset of triple-negative and, to a lower extent, HER2-positive breast cancers. It is also worth noting that we were able to relate ANXA1 expression to basal-like breast cancer as shown by a positive correlation with basal cytokeratin CK5/6. Again, this supports a similar finding by Yom et al. [21] who demonstrated that ANXA1 expression was significantly correlated with unfavorable prognostic factors such as hormone receptor negativity, HER2-positive tumors and triple-negative breast cancer. To our surprise though, Wang et al. [17] found that low ANXA1 expression was significantly associated with advanced stage breast cancer and a worse overall survival when compared to patients with high ANXA1 expression. The fact that an enhanced expression of ANXA1 was found in the so-called normal-like breast cancer subtype supports the view that this molecular category may simply reflect the high degree of contaminating normal breast tissue [35] and hence residual myoepithelial cells in the tumor samples. Future experiments with preclinical models will help solving this issue and/or this apparent contradiction 
Citation: Yousef EM, Laperrière D, Ramzan-Tahir M, Mader S, Gaboury LA (2013) Deregulated Expression of ANXA1 in Human High-Grade Breast Cancers. J Mol Biomark Diagn 4: 155. doi:10.4172/2155-9929.1000155

Page 9 of 10

Concerning the putative role of ANXA1 in the EMT, in this study we found a positive correlation between ANXA1 and VIM, one of EMT markers. Supporting data came from both the mRNA level using insilico analysis and from ANXA1 cell labeling on the TMAs. This may suggest that ANXA1 exerts a positive effect in EMT, consistent with reports indicating that ANXA1 promotes metastasis formation in basal-like breast cancer cells by enhancing TGF $\beta /$ smad signaling and actin reorganization [20]. Likewise, ANXA1-null mice were found to develop fewer metastases than wild type littermates [19]. However tantalizing this hypothesis might be, it is severely challenged in view of the findings by Maschler et al. [18] who demonstrated that forced ANXA1 expression in metastatic mouse and human mammary carcinoma cells reversed EMT and abolished metastasis. Clearly, more work is needed to sort out the exact role of ANXA1 in tumor progression and metastasis. The use of a comprehensive panel of EMT markers on human breast tumors expressing ANXA1 could help clarifying the issue. Of note, MSN and LYN that are known to play roles in EMT [36,37] have also positive correlation with ANXA1 (For MSN $r=0.69$, for LYN $r=0.54$ ) (Figure 6)

Lastly, a negative correlation between ANXA1 and both ESR1 and FOXA1 has been uncovered using in-silico study. This was further substantiated using a large cohort of breast cancer patients tissues and cell lines. $100 \%$ of patients with luminal breast cancer subtypes had positive expression of ESR 1 and FOXA1 while they completely lack ANXA1 expression. In contrast, $43 \%$ of triple negative breast cancers that are ESR1 and FOXA1 negative, display high levels of ANXA1. Recently, FOXA1 was reported to be necessary not only for maintaining luminal-specific gene expression, but also for repressing numerous genes specific to basal breast cancer cells including ANXA1 [38]. Interestingly, our in-silico study confirmed that ANXA1 promoter contains FOXA1 responsive element (two regions within 5kb of ANXA1 transcription start site) but none for ESR1. Given the suppressive roles of FOXA1 [39-41], it would be of great interest to determine how FOXA1 suppresses ANXA1 expression.

Lastly, one has to remind that ANXA1 is only one member of a broad family of annexins many of which have been reported to be directly or indirectly involved in breast cancer tumorigenesis such as Annexin A2, A4, A5 [23], A6 [32], A7 [2], A8 [42]. At present, the degree of functional overlap and crosstalk between each annexin variant has not been addressed.

To conclude, ANXA1 expression is restricted to high-grade breast cancers, mostly triple-negative breast tumors that are notoriously known for their aggressive clinical behavior. We propose that ANXA1 is deregulated in a significant proportion of high histological grade breast cancers, thereby underlining the complexity and heterogeneity of breast cancers especially those that belong to the triple-negative category. Developing new biomarkers will help dissect out subsets of triple-negative tumors and facilitate the identification and further characterization of unique and specific regulatory pathways paving the way to targeted therapy. A better understanding of the complex crossregulatory networks between ANXA1, Vimentin, ESR1 and FOXA1 signaling pathways is clearly needed to pinpoint and identify subsets of tumors with non-overlapping and unique mechanisms leading to tumor progression and metastasis.

\section{Acknowledgment}

This work was supported by Institute for Research in Immunology and Cancer (IRIC) departmental funding, Faculty of Graduate and Postdoctoral Studies of University of Montreal and The Egyptian Supreme Council of Universities.

\section{References}

1. Geyer FC, Rodrigues DN, Weigelt B, Reis-Filho JS (2012) Molecular classification of estrogen receptor-positive/luminal breast cancers. Adv Anat Pathol 19: 39-53

2. Desantis C, Ma J, Bryan L, Jemal A (2013) Breast cancer statistics, CA Cancer $\mathrm{J}$ Clin

3. Perez EA, Moreno-Aspitia A, Aubrey Thompson E, Andorfer CA (2010) Adjuvant therapy of triple negative breast cancer. Breast Cancer Res Treat 120: 285-291.

4. He J, Whelan SA, Lu M, Shen D, Chung DU, et al. (2011) Proteomic-based biosignatures in breast cancer classification and prediction of therapeutic response. Int J Proteomics: 896476.

5. Gabrovska PS, Robert Anthony, Haupt Larisa, Griffiths Lyn (2010) Gene Expression Profiling in Human Breast Cancer-Toward Personalised Therapeutics? The Open Breast Cancer Journal 2: 46-59.

6. Lim LH, Pervaiz S (2007) Annexin 1: the new face of an old molecule. FASEB J 21: 968-975.

7. Ang EZ, Nguyen HT, Sim HL, Putti TC, Lim LH (2009) Annexin-1 regulates growth arrest induced by high levels of estrogen in MCF-7 breast cancer cells. Mol Cancer Res 7: 266-274.

8. Babbin BA, Lee WY, Parkos CA, Winfree LM, Akyildiz A, et al. (2006) Annexin regulates SKCO-15 cell invasion by signaling through formyl peptide receptors. J Biol Chem 281: 19588-19599.

9. Hsiang CH, Tunoda T, Whang YE, Tyson DR, Ornstein DK (2006) The impact of altered annexin I protein levels on apoptosis and signal transduction pathways in prostate cancer cells. Prostate 66: 1413-1424.

10. Hu N, Flaig MJ, Su H, Shou JZ, Roth MJ, et al. (2004) Comprehensive characterization of annexin I alterations in esophageal squamous cell carcinoma. Clin Cancer Res 10: 6013-6022.

11. Garcia Pedrero JM, Fernandez MP, Morgan RO, Herrero Zapatero A, Gonzalez MV, et al. (2004) Annexin A1 down-regulation in head and neck cancer is associated with epithelial differentiation status. Am J Pathol 164: 73-79.

12. Xin W, Rhodes DR, Ingold C, Chinnaiyan AM, Rubin MA (2003) Dysregulation of the annexin family protein family is associated with prostate cancer progression. Am J Pathol 162: 255-261.

13. Bai XF, Ni XG, Zhao P, Liu SM, Wang HX, et al. (2004) Overexpression of annexin 1 in pancreatic cancer and its clinical significance. World $\mathrm{J}$ Gastroenterol 10: 1466-1470.

14. de Coupade C, Gillet R, Bennoun M, Briand P, Russo-Marie F, et al. (2000) Annexin 1 expression and phosphorylation are upregulated during liver regeneration and transformation in antithrombin III SV40 T large antigen transgenic mice. Hepatology 31: 371-380

15. Sinha P, Hütter G, Köttgen E, Dietel M, Schadendorf D, et al. (1998) Increased expression of annexin I and thioredoxin detected by two-dimensional gel electrophoresis of drug resistant human stomach cancer cells. J Biochem Biophys Methods 37: 105-116.

16. Perou CM, Sørlie T, Eisen MB, van de Rijn M, Jeffrey SS, et al. (2000) Molecular portraits of human breast tumours. Nature 406: 747-752.

17. Wang LP, Bi J, Yao C, Xu XD, Li XX, et al. (2010) Annexin A1 expression and its prognostic significance in human breast cancer. Neoplasma 57: 253-259.

18. Maschler S, Gebeshuber CA, Wiedemann EM, Alacakaptan M, Schreiber M et al. (2010) Annexin A1 attenuates EMT and metastatic potential in breast cancer. EMBO Mol Med 2: 401-414.

19. Yi M, Schnitzer JE (2009) Impaired tumor growth, metastasis, angiogenesis and wound healing in annexin A1-null mice. Proc Natl Acad Sci U S A 106 17886-17891.

20. de Graauw M, van Miltenburg MH, Schmidt MK, Pont C, Lalai R, et al. (2010) Annexin A1 regulates TGF-beta signaling and promotes metastasis formation of basal-like breast cancer cells. Proc Natl Acad Sci U S A 107: 6340-6345.

21. Yom CK, Han W, Kim SW, Kim HS, Shin HC, et al. (2011) Clinical significance of annexin A1 expression in breast cancer. J Breast Cancer 14: 262-268.

22. Jézéquel $P$, Frénel JS, Campion L, Guérin-Charbonnel $C$, Gouraud W, et al. (2013) bc-GenExMiner 3.0: new mining module computes breast cancer gene expression correlation analyses. Database (Oxford) 2013: bas060. 
Citation: Yousef EM, Laperrière D, Ramzan-Tahir M, Mader S, Gaboury LA (2013) Deregulated Expression of ANXA1 in Human High-Grade Breast Cancers. J Mol Biomark Diagn 4: 155. doi:10.4172/2155-9929.1000155

23. Neve RM, Chin K, Fridlyand J, Yeh J, Baehner FL, et al. (2006) A collection of breast cancer cell lines for the study of functionally distinct cancer subtypes. Cancer Cell 10: 515-527.

24. Miller LD, Smeds J, George J, Vega VB, Vergara L, et al. (2005) An expression signature for $\mathrm{p} 53$ status in human breast cancer predicts mutation status, transcriptional effects, and patient survival. Proc Natl Acad Sci U S A 102 13550-13555.

25. Cancer Genome Atlas Network (2012) Comprehensive molecular portraits of human breast tumours. Nature 490: 61-70.

26. Tavassoli FA DP (2003) World Health Organization classification of tumours. In Pathology and Genetics Tumours of the Breast and Female Genital Organs. Lyon: IARC Press : 19-23.

27. Hammond ME, Hayes DF, Wolff AC, Mangu PB, Temin S (2010) American society of clinical oncology/college of american pathologists guideline recommendations for immunohistochemical testing of estrogen and progesterone receptors in breast cancer. J Oncol Pract 6: 195-197.

28. Wolff AC, Hammond ME, Schwartz JN, Hagerty KL, Allred DC, et al. (2007) American Society of Clinical Oncology/College of American Pathologists guideline recommendations for human epidermal growth factor receptor 2 testing in breast cancer. Arch Pathol Lab Med 131: 18-43.

29. Caldarella A, Puliti D, Crocetti E, Bianchi S, Vezzosi V, et al. (2013) Biological characteristics of interval cancers: a role for biomarkers in the breast cancer screening. J Cancer Res Clin Oncol 139: 181-185

30. Subik K, Lee JF, Baxter L, Strzepek T, Costello D, et al. (2010) The Expression Patterns of ER, PR, HER2, CK5/6, EGFR, Ki-67 and AR by Immunohistochemical Analysis in Breast Cancer Cell Lines. Breast Cancer (Auckl) 4: 35-41.

31. Jonsson G, Staaf J, Olsson E, Heidenblad M, Vallon-Christersson J, et al (2007) High-resolution genomic profiles of breast cancer cell lines assessed by tiling BAC array comparative genomic hybridization. Genes Chromosomes Cancer 46: 543-58.
32. Sorlie T, Tibshirani R, Parker J, Hastie T, Marron JS, et al. (2003) Repeated observation of breast tumor subtypes in independent gene expression data sets. Proc Natl Acad Sci U S A 100: 8418-8423.

33. Elshimali Y, Liu P (2006) Expression of Annexin A1 in Normal and Malignan Breast Epithelium and Its Clinical Significance. Calcium Binding Proteins 1 : $51-53$

34. Kang H, Ko J, Jang SW (2012) The role of annexin A1 in expression of matrix metalloproteinase- 9 and invasion of breast cancer cells. Biochem Biophys Res Commun 423: 188-194.

35. Creighton CJ (2012) The molecular profile of luminal B breast cancer. Biologics 6: 289-297.

36. Wang CC, Liau JY, Lu YS, Chen JW, Yao YT, et al. (2012) Differential expression of moesin in breast cancers and its implication in epithelial-mesenchymal transition. Histopathology 61: 78-87.

37. Choi YL, Bocanegra M, Kwon MJ, Shin YK, Nam SJ, et al. (2010) LYN is a mediator of epithelial-mesenchymal transition and a target of dasatinib in breast cancer. Cancer Res 70: 2296-2306.

38. Bernardo GM, Bebek G, Ginther CL, Sizemore ST, Lozada KL, et al. (2013) FOXA1 represses the molecular phenotype of basal breast cancer cells. Oncogene 32: 554-563.

39. Cirillo LA, Lin FR, Cuesta I, Friedman D, Jarnik M, et al. (2002) Opening of compacted chromatin by early developmental transcription factors HNF3 (FoxA) and GATA-4. Mol Cell 9: 279-289.

40. Nakshatri H, Badve S (2009) FOXA1 in breast cancer. Expert Rev Mol Med 11: e8.

41. Robinson JL, Carroll JS (2012) FoxA1 is a key mediator of hormonal response in breast and prostate cancer. Front Endocrinol (Lausanne) 3: 68.

42. Stein T, Price KN, Morris JS, Heath VJ, Ferrier RK, et al. (2005) Annexin A8 is up-regulated during mouse mammary gland involution and predicts poor survival in breast cancer. Clin Cancer Res 11: 6872-6879. 